BULL. AUSTRAL. MATH. SOC.

VOL. 27 (1983), 157-158.

\title{
CONTINENTAL SHELF DYNAMICS
}

\author{
Ian LesLie Collings
}

It is now well known that a continental slope or shelf can act as a wave guide for the uni-directional propagation of low frequency energy. Such motions are cormonly referred to as continental shelf waves.

With the notable exception of $\mathrm{Ni}$ iler and Mysak [1], most theories of continental shelf waves have ignored the effect of a mean coastal current. The effects of such a mean current are the advection of the shelf wave modes and the consequent modification of their propagation characteristics, the introduction of a new class of shear wave (in the case of a barotropic current), and the possibility of instabilities due to the lateral and/or vertical shear of the current. Such instabilities may manifest themselves as meanders or eddies, the presence of which in the world's major currents being now well documented.

In this study a theoretical investigation of instabilities associated with sub-inertial barotropic motions propagating along a straight continental shelf in the presence of a longshore mean current is undertaken. The motion is assumed to take place on an $f$-plane and the appropriate linearised shallow-water barotropic equations are derived assuming the non-divergent approximation. Some general results on stability are presented and sufficient conditions for stability derived; possible discontinuities in the current shear and the depth profile are permitted. Asymptotic expansions for both long and short. waves are described.

Analytic solutions are obtained for an extensive range of cases where

Received 5 November 1982. Thesis submitted to University of Melbourne, June 1982. Degree approved, October 1982. Supervisor: $\mathrm{Dr}$ R.H.J. Grimshaw. 
the topography is piece-wise constant and the current piece-wise linear. Two kinds of instability, a shear instability and a topographic instability, are identified. Analytic solutions are also obtained when the current is piece-wise linear but the depth profile exponential. The results so obtained are applied to models of the East Australian Current, the Gaspe Current and the Gulf Stream.

To estimate the effects of stratification, a two layer model is considered and a perturbation scheme developed for the piece-wise constant depth profile and the piece-wise linear current profile; the stratification is taken to be "on shelf" and it is assumed that there is no vertical shear between the two layers. Internal Kelvin waves trapped about discontinuities in the current shear are identified.

The effect of a mean current with transverse shear on topographic Rossby waves is considered with particular application to the East Australian shelf. The modifications due to a linear friction law are also considered and the resultant predicted phase lag and inshore current velocities are shown to be in good agreement with observations.

\section{Reference}

[1] Pearn P. Ni iler and Lawrence A. Mysak, "Barotropic waves along an eastern continental shelf", Geophys. Fluid Dyn. 2 (1971), 273-288.

Division of Computing and Mathematics, Deakin University, victoria 3217 , Australia. 